Gabrielle Angela G. Mercado, MD

Kirt Areis E. Delovino, MD

Allan B. Carpela, MD

Department of Otolaryngology

Head and Neck Surgery

St. Luke's Medical Center, Quezon City

\section{Arteriovenous Malformation of the Mandible in a Young Postpartum Woman}

\begin{abstract}
Objective: To present a case of arteriovenous malformation of the mandible in a young postpartum woman and discuss the diagnostic and therapeutic dilemmas involved.

Methods:

$\begin{array}{ll}\text { Design: } & \text { Case Report } \\ \text { Setting: } & \text { Tertiary Private Teaching Hospital } \\ \text { Participants: } & \text { One }\end{array}$

Result: A 27-year-old woman with left mandibular swelling two weeks after tooth extraction was diagnosed and managed as a case of arteriovenous malformation. The extent of the lesion prevented surgical intervention and bleeding recurred despite three embolization attempts. Ultimately, the left external carotid artery and contralateral dorsal contributory vessel were sacrificed causing necrosis of the anterior tongue and its eventual detachment. Three sessions of stereotactic radiosurgery eventually controlled the bleeding with no recurrence to date.
\end{abstract}

Conclusion: On her second month following stereotactic radiosurgery, there appeared to be no complete resolution of the AVM but there was also no recanalization or regrowth of the lesion. Uncertain about whether her arteriovenous malformation will revascularize and how we may be able to help her in the event that it does, the waiting game continues for our patient.

\section{Keywords: arteriovenous malformations; mandible, abnormalities; vascular malformations}

Arteriovenous malformations (AVM) of the mandible account for 1\% of facial AVM and represent the most severe vascular conditions in the head and neck presenting with pain, loose teeth, gum bleeding, swelling, pulsations and a thrill or bruit.' Symptoms of mandibular vascular malformations may be subtle and related to a preceding dental surgical procedure as previously reported for mandibular hemangiomas. ${ }^{2,3}$ However, to the best of our knowledge, there are no previous local reports of AVM in the mandible. We report a case of AVM of the mandible in a young postpartum woman and discuss the diagnostic and therapeutic dilemmas involved.

\section{CASE REPORT}

A 27-year-old woman presented with painless left mandibular swelling two weeks after extraction of the left second and third mandibular molar due to loose dentition. She narrated that heavy bleeding had been encountered during the tooth extraction but was controlled with no postoperative bleeding episodes. Healing of the operative site was unremarkable and completed within 2 weeks. The patient was initially treated with oral antibiotics, but the left mandibular swelling persisted and a faint intermittent throbbing sensation was felt along the left postauricular area. She was then referred to an ear, nose, throat (ENT) surgeon for further evaluation and management. 
A Computed Tomography (CT) scan revealed soft tissue attenuation in the medullary portion of the body and ramus of the left side of the mandible causing destruction of the surrounding bone and cortex with irregular areas of lucency. (Figure 1) Because of the non-painful mandibular swelling after tooth extraction and CT scan findings, the primary consideration was an odontogenic tumor probably an ameloblastoma. A segmental mandibulectomy was recommended but she opted to obtain a second opinion at our institution. On examination, the swelling was only noticeable externally with a very faint area of hyperemic skin overlying the left mandibular body and no facial asymmetry, pain or numbness. She was admitted for biopsy and possible left marginal mandibulectomy. Upon incising along the left retromolar trigone to the area of the left lower second molar, profuse bleeding amounting to $500 \mathrm{ml}$ was encountered. Hemostasis was done and the procedure was aborted pending further diagnostic work-ups.

Magnetic Resonance Angiography (MRA) of the oral cavity revealed multiple serpiginous flow void signals seen in the region of the left mandible with cortical erosion and intraosseous involvement as well as involvement of the masseter muscles, masticator and parapharyngeal spaces, with communication with the carotid vessels. (Figure 2) A vascular malformation was confirmed by bilateral carotid and facial angiography. (Figure 3) The image showed a moderate to high-flow vascular malformation involving the left facial, mandibular and auricular areas with prominent hypertrophied arterial supplies mainly coming from bilateral lingual and facial arteries, the left internal maxillary and pre-auricular arteries, and drained by the corresponding markedly dilated veins in the left side.

From the initial admission, our patient was readmitted three more times due to recurrence of oral cavity bleeding despite repeated permanent embolization. The severity of oral cavity bleeding worsened with each admission. The patient experienced episodes of hypovolemic shock, loss of consciousness and seizures. The AVM was seen to extend intracranially with recanalizaton of the previously embolized blood vessels arising from the left lingual and left facial arteries with the right facial and right lingual arteries more engorged, with progression in degree of collateral vessel formation. The descending branch of the right internal maxillary artery now contributed to the AVM along the left mandible. (Figure 4) The patient was being seen by a multispecialty services to discuss the possible options and risks that the patient could encounter.

The possibility of performing the mainstay treatment of AVM involving embolization and complete excision was not a primary consideration at this point because of the high likelihood of mortality. She was referred to vascular surgery for possible clipping of vessels, radiation oncology for stereotactic radio surgery, endovascular or external beam radiation and interventional oncology for possible reembolization. The chance of complete devascularization of the main

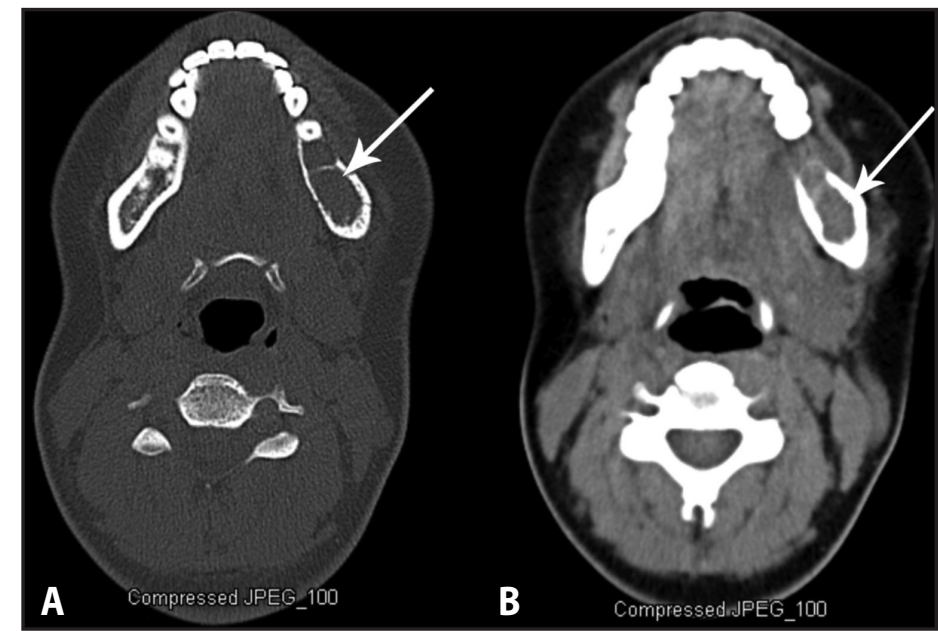

Figure 1. Axial CT Scans at the level of the mandible A. bone window, B. soft tissue window showing soft tissue attenuation in the medullary portion of the body and ramus of the left side of the mandible causing destruction of the surrounding bone and cortex resulting in irregular areas of lucency (arrows).

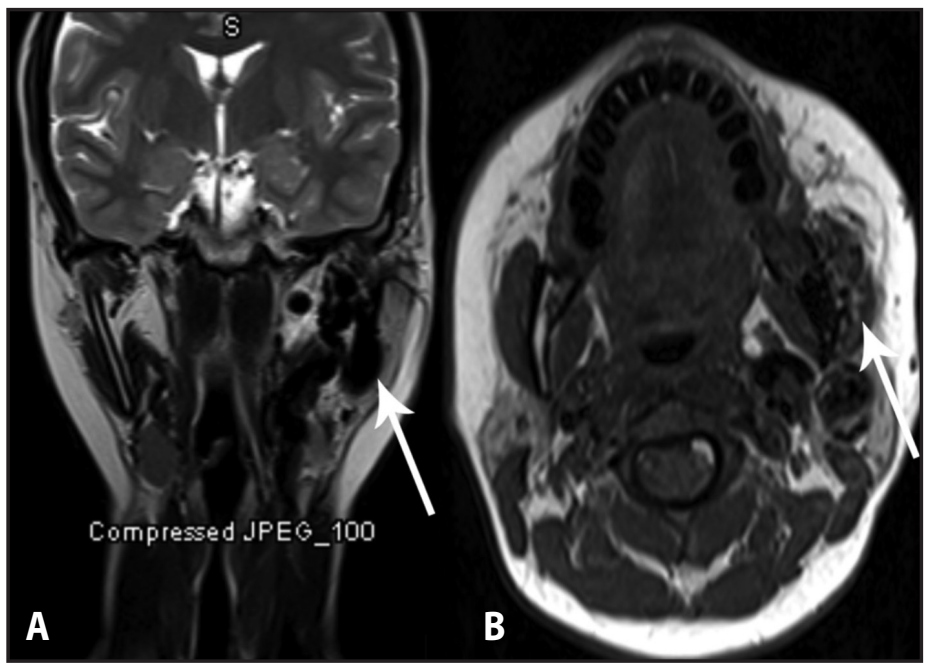

Figure 2. MRA of the oral cavity A. coronal view at the level of the parapharyngeal space. B. axia view at the angle of mandible. Multiple serpiginous flow void signals are seen in the region of the left mandible with cortical erosion and intraosseous involvement as well as involvement of the masseter muscles, masticator and parapharyngeal spaces and communication with the carotid vessels (arrows).

branches of the carotid artery was highly considered, but so was the possible compromise of blood supply to the tongue and mandible leading to eventual necrosis.

After losing 1.5 liters of blood from the dental sockets within just a few minutes during the third admission, vascular ligation or complete extirpation of the AVM was no longer considered an option due to the likelihood of intractable blood loss and necrosis. The most feasible management considered was selective catheterization of the prominent hypertrophied arterial supplies arising from both external carotid arteries and polyvinyl alcohol embolization that resulted in complete devascularization of the said vessels. She was discharged stable, but reported occasional left mandibular pain and progression of skin discoloration along the left mandible with necrosis of the anterior 


\section{CASE REPORTS}

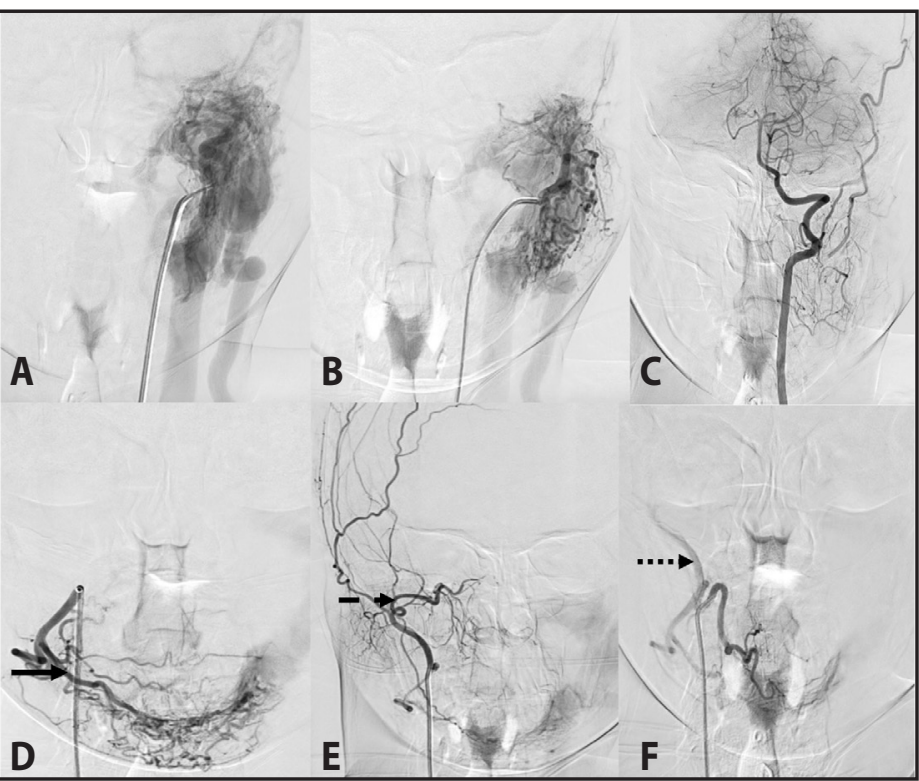

Figure 3. Serial $C T$ angiogram of carotid arteries, $L$ to $R$, top to bottom. Moderate to high-flow vascular malformation involving the left facial, mandibular and auricular areas with prominent hypertrophied arterial supplies were mainly coming from bilateral lingual and facial arteries (solid arrows), and left internal maxillary (dashed arrows) and pre-auricular arteries (dotted arrows).

\section{third of the tongue. (Figure 5A)}

Stereotactic surgery was believed to be the only other option for our patient. On the 49th day, the patient received her first session of stereotactic surgery. On the same day, her necrotic anterior tongue fell off. (Figure 5B) She completed stereotactic surgery sessions on the 55th day. Monthly follow ups since have shown no significant interval change in degree and extent of the previously dilated and tortuous varices in the left retromandibular region, and no new formation or recanalization of the arteriovenous malformation. (Figure 6) Although the patient had lost the anterior third of her tongue with resulting dysarthria, her mandible was still intact with no progression of skin changes and she was able to tolerate a pureed diet. Until this writing, there has been no recurrence of bleeding and our patient has been undergoing speech and swallowing therapy and vital stimulation.

\section{DISCUSSION}

Arteriovenous malformations, a subtype of vascular malformations are congenital malformations accounting for $1.5 \%$ of all vascular anomalies with $70 \%$ involving the cheek, nose, ears and upper lip. Exemplified by our patient, more females than males (ratio of 1.5:1) present with AVM that commonly involves the cheek. ${ }^{4}$ These tumors result from errors of vascular morphogenesis that are caused by a disturbance in the late stages of angiogenesis. ${ }^{1}$

Mandibular AVM are rare in clinical practice, and are often found by chance when teeth in the area are extracted ${ }^{5}$ as was the case with our patient. Patients are usually pediatric but may not become symptomatic until later in life when they present with facial swelling, pain, otalgia, dental mobility or spontaneous bleeding around the teeth or from the mucosa. ${ }^{6}$ Having a stage 1 AVM according to the Schobinger classification, ${ }^{7}$ our patient developed symptoms (including minimal nontender facial swelling and a vague pulsation felt along the cheek) later on in life. According to Su et al. AVM expand rapidly from an idle state especially after exposure to trauma during pubescence or following the change of estrogenic hormones such as when one is pregnant. ${ }^{5}$ Our patient had recently given birth when she began to notice mandibular skin discoloration.

Vascular malformations are not known to resolve spontaneously and can be classified further according to hemodynamic criteria into high-flow (AVMs, arteriovenous fistulae) or low flow (capillary, venous, hemangiomas and lymphatic malformations) lesions.,9 High-flow vascular malformations are commonly located along the cheek or auricle

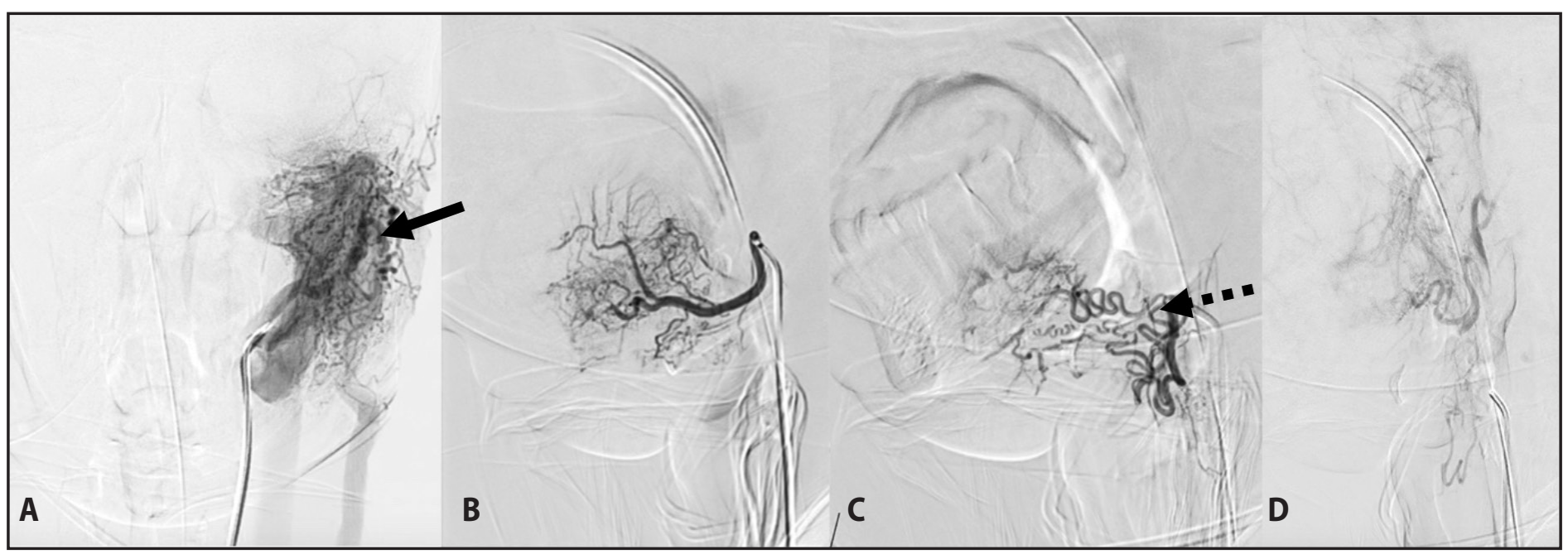

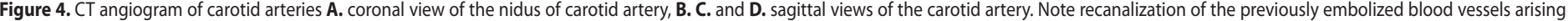

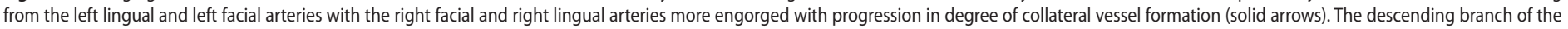
right internal maxillary artery now appears to contribute to the left mandibular arteriovenous malformation (dotted arrows). 


\section{CASE REPORTS}

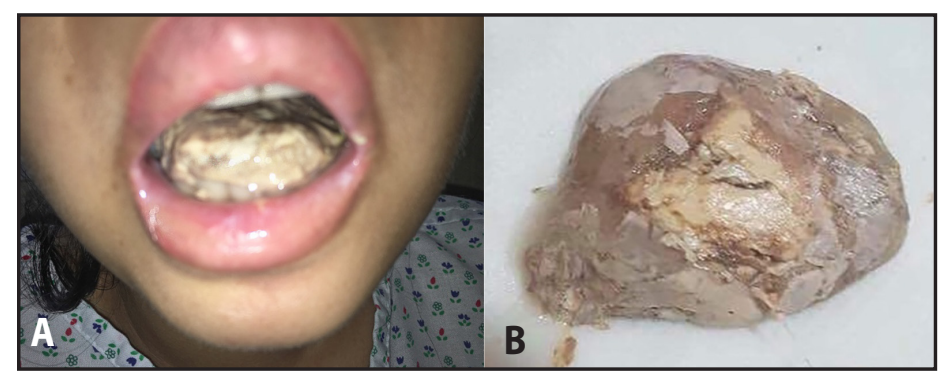

Figure 5. Appearance of tongue after complete embolization of the carotid vessels $\mathbf{A}$. Necrotic anterior third of the tongue B. Auto-amputated anterior third of the tongue.

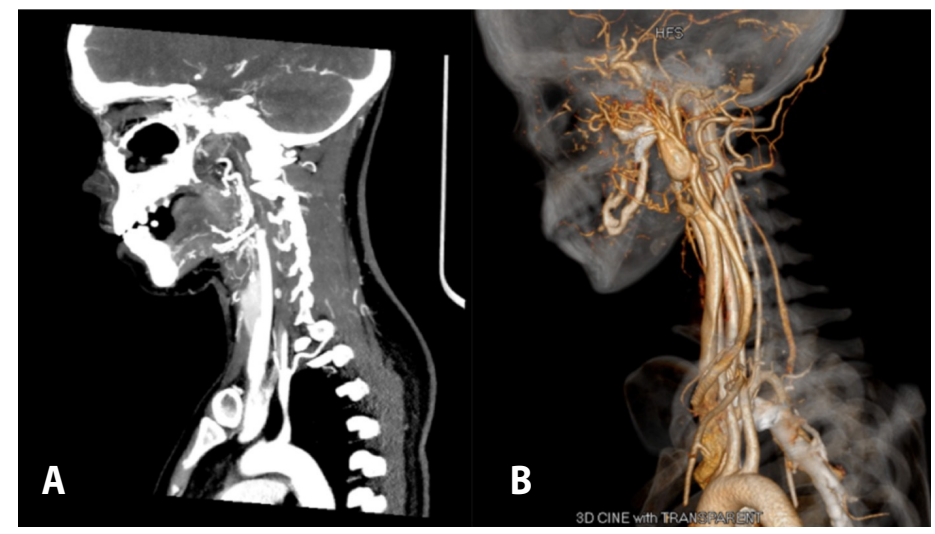

Figure 6. CT angiogram of carotid arteries, taken after stereotactic radiosurgery. A. and B. No significant interval change in degree and extent and no new formation or recanalization of the arteriovenous malformation.

as localized pulsatile masses or diffuse areas of increased blood flow. ${ }^{9}$

There is no single definitive treatment for AVM. Options include conservative management (transvenous or transarterial embolization, radiotherapy, sclerotherapy, radiotherapy, bone wax packing of bone cavities and curettage), surgical resection and reconstruction or a combination of both. ${ }^{1,6}$ Studies have shown that a single session of superselective intra-arterial embolization is enough to control small high flow vascular malformations of the oral cavity with postprocedural follow up. ${ }^{10,11}$ Small AVMs including those considered high-flow can be treated by surgical excision and embolization, but this may not apply to large AVMs such as that in our patient due to the increased risk of exsanguination and incomplete extirpation of the nidus. Considering the patient's petite stature, the margin for blood loss was very low and multiple episodes of oral cavity bleeding almost led to exsanguination. Our patient presented with a large high-flow vascular malformations and recurrent profuse bleeding. Ligation of the carotid artery and surgical excision was not considered appropriate management as it could not be completely embolized or resected. We resorted to serial embolization, complications of which may include hemorrhage, hematoma formation, infection, dissecting aneurysm, cerebrovascular accident, cranial nerve injury, facial paralysis, skin and mucosal necrosis and ultimately, death. ${ }^{12,13}$ Not only was embolization insufficient, the AVM in our patient could not be controlled despite multiple embolization attempts that eventually led to tongue necrosis and auto- amputation, permanently affecting her quality of life.

The medical team was uncertain about the proper management for our patient. Partial and complete embolization of the external carotid artery to control the nidus was attempted but proved to be insufficient. Complete surgical resection was not considered an option due to the high risk of exsanguination and extreme facial deformity. There is no definitive management guideline for such a case. Had the patient presented with a soft tissue AVM, the chances of diagnosis and treatment would have been higher compared to the intraosseous AVM which can present as an apparently normal or asymptomatic lesion.

Our patient has received three sessions of embolization and stereotactic surgery. She has already lost her ability to speak and has impaired feeding and swallowing. On her second month following stereotactic radiosurgery, there appears to be no complete resolution of the AVM but there is also no recanalization or regrowth of the lesion. Uncertain about whether her arteriovenous malformation will revascularize and how we may be able to help her in the event that it does, the waiting game continues for our patient.

\section{ACKNOWLEDGEMENTS}

We thank Dr. Joel A. Romualdez, Joanne Sebastiana M. De Ramos, Dr. Marcelino S. Tuaño and Dr. Jimmy V. Chang for the suggestions and comments regarding the focus and flow of the case report. We also thank our chairs Dr. Cecilia Gretchen Navarro-Locsin (QC) and Dr. William Lim (BGC) and Residency Training Officers Dr. Joseph Arañas (QC), and Dr. Keith Aguilera (BGC) for their continued support and encouragement in writing scientific papers.

\section{REFERENCES}

1. Loureiro CC, Falchet PC, Gavranich J Jr, Lobo Leandro LF. Embolization as the treatment for a life-threatening mandibular arteriovenous malformation. J Craniofac Surg. 2010 Mar;21(2): 380382. DOI: 10.1097/SCS.0b013e3181cfa62a; PMID: 20186078

2. Papa EC, Samson ES, Victoria FA. Cavernous hemangioma of the mandible. Philipp J Otolaryngol Head Neck Surg. 2009;24(2):32-35. DOI: 10.32412/pjohns.v24i2.685.

3. Sadain-Urao ZK, Pontejos AQY. Hemangioma of the mandible: An exanguinating lesion. Philipp J Otolaryngol Head Neck Surg. 1998:21-26. [cited 2019 May 26] Available from: https://pjohns. pso-hns.org/index.php/pjohns/issue/view/73/21.

4. Shailaja SR, Manika M, Manjula M, Kumar LV. Arteriovenous malformation of the mandible and parotid gland. Dentomaxillofac Radiol. 2012 Oct; 41(7): 609-614. DOI: 10.1259/dmfr/47383305; PMID: 22282511 PMCID: PMC3608384.

5. Su LX, Fan XD, Zheng JW, Wang YA, Qin ZP, Wang XK, et al. A practical guide for diagnosis and treatment of arteriovenous malformations in the oral and maxillofacial region. Chin J Dent Res. 2014; 17(2):85-9. PMID: 25531015.

6. Wang J, Huang H. Intraoral curettage without presurgical endovascular embolization: a simple but controversial treatment of arteriovenous malformations of the mandible. Int J Oral Maxillofac Surg. 2013 Jan; 42(1): 133-136. DOI: 10.1016/j.ijom.2012.04.015; PMID: 22608197.

7. Mulligan PR, Prajapati HJ, Martin LG, Patel TH. Vascular anomalies: classification, imaging characteristics and implications for interventional radiology treatment approaches. Br J Radiol. 2014 Mar;87(1035):20130392. DOI: 10.1259/bjr.20130392 PMID: 24588666. PMCID:PMC4064609

8. Ayahao F. Feeding and draining vessel ligation with sclerotherapy of high flow arteriovenous malformations in the head and neck. Philipp J Otolaryngol Head Neck Surg. 2014 Jan-Jun; 29(1) 37-40. DOI:https://doi.org/10.32412/pjohns.v29i1.467.

9. Kriwalsky MS, Papadimas D, Maurer P, Brinkmann M, Jackowski, J, Kunkel M. Life-threatening bleeding after tooth extraction due to vascular malformation: a case report and literature review. Oral Maxillofac Surg. 2014 Sep; 18(3): 279-82. DOI: 10.1007/s10006-014-0448-3; PMID 24756853.

10. Perkins J. Vascular Anomalies of the Head and Neck. In: Flint P, Haughey B, Lund V, Niparko J, Robbins T, Thomas R, et al., editors. Cummings Otolaryngology: Head and Neck Surgery 6th edition. Philadelphia, PA: Saunders Elsevier. 2015. p.3078.

11. Tarkan $\mathrm{O}$, Sürmelioğlu $\mathrm{O}$, Tuncer Ü, Akgül E. Face skin necrosis following embolization for arteriovenous malformations: a case report. Oral Maxillofac Surg. 2010 Mar; 14(1):49-52. DOI: 10.1007/s10006-009-0180-6; PMID: 19830463.

12. Corsten L, Bashir Q, Thornton J, Aletich V. Treatment of a giant mandibular arteriovenous malformation with percutaneous embolization using histoacrylic glue: a case report. $J$ Oral Maxillofac Surg. 2001 Jul; 59(7): 828-832. DOI: 10.1053/joms.2001.24311; PMID: 11429753.

13. Bouloux GF, Perciaccante VJ. Massive hemorrhage during oral and maxillofacial surgery: ligation of the external carotid artery or embolization? J Oral Maxillofac Surg. 2009 Jul; 67 (7):15471551. DOI: 10.1016/j.joms.2009.03.014; PMID: 19531434. 